\title{
Tétano: Um problema de saúde pública no Brasil apesar das estratégias e medidas de
}

\section{prevenção}

\author{
Tetanus: A public health problem in Brazil despite prevention strategies and measures \\ Tétanos: Un problema de salud pública en Brasil a pesar de las estrategias y medidas de prevención
}

Recebido: 09/04/2021 | Revisado: 15/04/2021 | Aceito: 19/04/2021 | Publicado: 04/05/2021

Eduarda Cardoso de Lima

ORCID: https://orcid.org/0000-0001-5103-5797

Universidade Federal de Santa Maria, Brasil

E-mail: eduarda.cardoso@acad.ufsm.com

Júlia Glowacki

ORCID: https://orcid.org/0000-0001-8860-3356

Universidade Federal de Santa Maria, Brasil

E-mail: glowacki.julia@gmail.com

Luisa Fontella Barroso

ORCID: https://orcid.org/0000-0001-8843-3924

Universidade Federal de Santa Maria, Brasil

E-mail: luisabarroso0062@gmail.com

Naiane Couto Fonseca

ORCID: https://orcid.org/0000-0002-6885-047X

Universidade Federal de Santa Maria, Brasil

E-mail: naiane.fonseca@ hotmail.com

Sabrina Zancan

ORCID: https://orcid.org/0000-0001-9219-1286

Universidade Federal de Santa Maria, Brasil E-mail: sabrina_zancan@yahoo.com.br

Andressa da Silveira

ORCID: https://orcid.org/0000-0002-4182-4714

Universidade Federal de Santa Maria, Brasil E-mail: andressadasilveira@gmail.com

\begin{abstract}
Resumo
Objetivo: Descrever o perfil epidemiológico dos casos notificados de Tétano acidental no Brasil entre os anos de 2009 e 2019. Metodologia: Trata-se de um estudo quantitativo descritivo, com dados obtidos na plataforma DataSUS, por meio da seguinte estratégia: "Informações de Saúde (TABNET)", no grupo "Doenças e Agravos de Notificação 2007 em diante (SINAN)", com destaque em "tétano". Foram aplicadas as seguintes variáveis: regiões do Brasil, ano de notificação (2009 a 2019), idade, sexo, escolaridade e evolução. Resultados: Os achados da pesquisam revelam que o sexo masculino representa $85,3 \%$ dos casos de TA e que a região Nordeste apresenta o maior percentual de notificações $(33,2 \%)$. Dos contaminados, $38,1 \%$ vieram a óbito. Os indivíduos com idade entre 20 e 59 anos representam $60,2 \%$ dos casos totais e aqueles que não completaram os estudos da $1^{\mathrm{a}}$ a $4^{\mathrm{a}}$ série do ensino fundamental representam 28,9\%. O ano de 2011 é o que mais apresentou casos confirmados de TA (335). Conclusão: Os resultados indicam que, mesmo com a queda dos números de casos, o TA ainda é um problema de saúde pública e apresenta uma taxa de letalidade significativa. Portanto, urge a necessidade de manter em dia o esquema de vacinação e ampliar o conhecimento dos brasileiros acerca das formas de prevenção da doença.
\end{abstract}

Palavras-chave: Enfermagem em saúde pública; Epidemiologia; Prevenção de doenças; Tétano.

\begin{abstract}
Objective: To describe the epidemiological profile of reported cases of accidental Tetanus in Brazil between the years 2009 and 2019. Methodology: This is a quantitative descriptive study, with data obtained in the DataSUS platform, through the following strategy: "Health Information (TABNET)", in the group "Diseases and Diseases of Notifications - 2007 onwards (SINAN)", with emphasis on "tetanus". The following variables were applied: regions of Brazil, year of notification (2009 to 2019), age, sex, education, and evolution. Results: The research findings reveal that the male sex represents $85.3 \%$ of the cases of accidental Tetanus and that the Northeast region presents the highest percentage of notifications (33.2\%). Of those contaminated, 38.1\% deceased. Individuals aged between 20 and 59 years represent $60.2 \%$ of the total cases and those who have not completed studies from the 1st to the 4th grade of elementary school represent $28.9 \%$. 2011 is the year with the most confirmed cases of accidental tetanus (335). Conclusion: The results indicate that, even with the drop in the number of cases, accidental Tetanus is still a public
\end{abstract}


health problem and has a high lethality rate. Therefore, there is an urgent need to keep the vaccination schedule up to date and expand the knowledge of Brazilians about ways of preventing the disease.

Keywords: Public health nursing; Epidemiology; Prevention of diseases; Tetanus.

\begin{abstract}
Resumen
Objetivo: Describir el perfil epidemiológico de los casos reportados de Tétanos accidental en Brasil entre los años 2009 y 2019. Metodología: Se trata de un estudio cuantitativo descriptivo, con datos obtenidos en la plataforma DataSUS, a través de la siguiente estrategia: "Información de Salude (TABNET)", en el grupo "Enfermedades y Enfermedades de Notificaciones - 2007 en adelante (SINAN)", con énfasis em "tétanos". Se aplicaron las siguientes variables: regiones de Brasil, año de notificación (2009 a 2019), edad, sexo, educación y evolución. Resultados: Los hallazgos de la investigación revelan que los hombres representan el 85,3\% de los casos de Tétanos accidental y que la región Nordeste tiene el mayor porcentaje de notificaciones (33,2\%). De los contaminados, el 38,1\% falleció. Los individuos con edades comprendidas entre los 20 y los 59 años representan el 60,2\% del total de casos y los que no han finalizado sus estudios del $1^{\circ}$ al $4^{\circ}$ grado de primaria representan el 28,9\%. 2011 es el año con más casos confirmados de Tétanos accidental (335). Conclusión: Los resultados indican que, incluso con la caída en el número de casos, el Tétanos accidental sigue siendo un problema de salud pública y tiene una alta tasa de letalidad. Conclusión: Por lo tanto, existe una necesidad urgente de mantener actualizado el calendario de vacunación y ampliar el conocimiento de los brasileños sobre las formas de prevenir la enfermedad.
\end{abstract}

Palabras clave: Enfermería de salud pública; Epidemiología; Prevención de enfermedades; Tétanos.

\title{
1. Introdução
}

O Tétano é uma doença infecciosa desencadeada a partir da ação da bactéria gram-positiva Clostridium tetani. O agente patogênico produz neurotoxinas que provocam a hiperexcitabilidade do sistema nervoso, causando espasmos musculares (Tortora et al., 2017). A doença pode ocorrer em todas as idades e, dependendo do modo de contágio, pode ser classificada como acidental ou materno neonatal (Organização Mundial da Saúde, 2017).

O tétano acidental (TA) acontece a partir do contato de ferimentos com a bactéria que na forma de esporos pode estar presente em parte do ambiente, como no solo. A contaminação com o agente também pode acontecer durante a realização de cirurgias e outros procedimentos em espaço não estéril. Quando a contaminação ocorre através de partos e abortos feitos nessas condições, a doença é denominada tétano materno. O tétano neonatal (TNN), por sua vez, ocorre especificamente em neonatos de até 28 dias de vida que têm contato com os esporos por meio de materiais mal higienizados, utilizados principalmente no manejo do cordão umbilical. A doença não é contagiosa, logo não ocorre transmissão de pessoa para pessoa (Organização Mundial da Saúde, 2017).

O período de incubação para o TA é de 5 a 15 dias. Os primeiros sintomas são a dificuldade de abertura da boca e a rigidez muscular. Progressivamente, a doença pode atingir outros grupos musculares, como torácicos, abdominais e inferiores. Se a infecção não é tratada, o paciente evolui para óbito em virtude da insuficiência respiratória. Desta forma, uma pessoa com sintomas de tétano deve procurar o serviço de saúde para realizar o diagnóstico, que é confirmado por meio de critério clínico e laboratorial (Ministério da Saúde, 2019).

$\mathrm{O}$ tratamento do tétano é baseado na redução dos sintomas, neutralização das toxinas, eliminação das bactérias e cuidados com a ferida. Contudo, deve ser realizado em ambiente hospitalar e, em casos mais graves, na Unidade de Tratamento Intensivo (UTI). Os cuidados promovem o relaxamento da musculatura, diminuindo a dor causada pelos espasmos e permitindo melhoras no padrão respiratório do paciente. A neutralização ocorre com soro antitetânico (SAT) ou com imunoglobulina humana antitetânica (IGHAT), promovendo a imunização passiva. Para a eliminação do agente patogênico são administrados antibióticos, como a penicilina e o metronidazol (Ministério da Saúde, 2019).

A forma mais segura de prevenir o tétano é por vacinas contendo o toxóide tetânico (VCCT). No Brasil, o Programa Nacional de Imunização (PNI) recomenda que a partir dos 2 meses de vida a criança receba 3 doses da vacina pentavalente, contra difteria, tétano, coqueluche, Haemophilus influenzae Tipo B e poliomielite, com intervalo de dois meses. Reforços de DTP, contra difteria, tétano e coqueluche, são programados para o $15^{\circ}$ mês e o $4^{\circ}$ ano de vida. A partir da adolescência é 
indicado receber uma aplicação a cada 10 anos, até a velhice, da vacina dupla adulta, Dt ou Td, contra tétano e difteria. No caso de mulheres grávidas é recomendada uma dose de dTpa, contra difteria, tétano e coqueluche como reforço. Caso a gestante não tenha histórico vacinal deve receber 3 doses da vacina dupla adulta (Ministério da Saúde, 2021).

Frente a situações em que há ferimento e não é confirmado o histórico vacinal, a pessoa é instruída a receber a vacina. Após a recuperação da infecção o paciente não adquire imunidade, podendo ser infectado outras vezes com o Clostridium tetani, assim é necessário vacinar-se com VCTT (Ministério da Saúde, 2019).

A partir da confirmação de um caso de TA é necessária a notificação ao Sistema de Informação de Agravos de Notificação (SINAN). Desde 1998, quando o SINAN passou a ser regulamentado, tornou-se obrigatório o fornecimento de notificações pelos municípios, estados e Distrito Federal para criação de um banco de dados nacional (Ministério da Saúde, 2007). Desta forma, espera-se que as notificações representem a realidade, apesar das subnotificações.

Embora seja uma doença imunoprevinível, o TA apresenta alta taxa de letalidade e se configura como um problema de saúde pública no Brasil (Ministério da Saúde, 2018). É visível, portanto, a importância da vacinação, bem como da disseminação das formas de prevenção.

Este estudo tem o objetivo de descrever o perfil epidemiológico dos casos notificados de Tétano acidental no Brasil durante o período de 2009 a 2019.

\section{Metodologia}

Trata-se de um estudo quantitativo descritivo, realizado a partir dos dados secundários obtidos pela plataforma Departamento de Informática do Sistema Único de Saúde (DataSUS). Para a coleta de dados foram realizados os seguintes passos no sistema: "Informações de Saúde (TABNET)", "Epidemiológicas e morbidade", no grupo "Doenças e Agravos de Notificação - 2007 em diante (SINAN)", com destaque em "tétano acidental", em todas as regiões brasileiras. Foram obtidas as seguintes variáveis: regiões do Brasil (Norte, Sul, Nordeste, Sudeste e Centro-Oeste), ano de notificação (2009 a 2019), idade (menos de 1 ano, 1 a 4 anos, 5 a 9 anos, 10 a 14 anos, 15 a 19 anos, 20 a 39 anos, 40 a 59 anos, 60 a 64 anos, 65 a 69 anos, 70 a 79 anos e 80 anos e mais), sexo (feminino e masculino), escolaridade (analfabetos, $1^{\mathrm{a}}$ a $4^{\mathrm{a}}$ série incompleta do ensino fundamental, $4^{\mathrm{a}}$ série completa do ensino fundamental, $5^{\mathrm{a}}$ a $8^{\mathrm{a}}$ série incompleta do ensino fundamental, ensino fundamental completo, ensino médio incompleto, ensino médio completo, educação superior incompleta e educação superior completa) e evolução (cura e óbito). A análise estatística dos dados ocorreu por meio do Excel.

Este estudo envolveu o levantamento de informações originadas de banco de dados de uso e acesso público DataSUS, o que justifica a ausência da apreciação de um Comitê de Ética, em conformidade com a Resolução nº 510/2016, em que não é necessário registrar no Comitê de Ética e Pesquisa com seres humanos aqueles estudos que utilizam dados de acesso público, domínio público e/ou que estejam em banco de dados sem possibilidade de identificação individual.

\section{Resultados e Discussão}

No período de 2009 a 2019 foram notificados o total de 2.984 casos de TA no Brasil. A Figura 1 descreve os casos estratificados por região de notificação e sexo. 
Figura 1. Casos notificados de Tétano acidental no Brasil por região de notificação e sexo no período de 2009 a 2019.



Fonte: Dados da pesquisa (2020).

Em relação a variável região, o Nordeste apresentou o maior registro de casos (33,3\%), seguido do Sudeste $(22,4 \%)$, Sul (19,8\%), Norte (12,9\%) e Centro-Oeste (11,5\%). A média de casos notificados por região foi de 596,8, com desvio padrão de 260,7 .

O Nordeste apresentou crescimento econômico e expandiu o acesso da população à serviços diversos. Essa "política de expansão", porém, se concentrou nas capitais e polos regionais, de forma que parte significativa do corpo social foi marginalizada e continuou a lidar com a inacessibilidade de alguns serviços, dentre os quais está o atendimento em saúde (Albuquerque et al., 2017). Quando aplicado à população de baixo status econômico que depende apenas do Sistema Único de Saúde (SUS), o acesso aos serviços de saúde se torna ainda mais escasso (Dantas et al., 2021). Esse cenário deixa a população da região desamparada e suscetível a doenças como o TA.

Quanto à variável sexo, foi observado que 85,3\% dos casos notificados são de pessoas do sexo masculino, enquanto 14,7\% são de pessoas do sexo feminino. Ao verificar ambas as estratificações (por região e por sexo), observou-se que a porcentagem de homens infectados por região varia de $81 \%$ a 90,6\%. Desta forma, os homens são os mais afetados pela doença, independentemente da região de residência.

A predominância da doença sobre o sexo masculino pode estar associada às atividades profissionais realizadas pelos homens. Um estudo feito com trabalhadores de uma empresa em Santa Cruz do Sul (RS) apontou que indivíduos do sexo masculino apresentam menor adesão ao esquema de imunização ocupacional e que a dT está entre as vacinas com baixo índice de funcionários imunizados (Hiester, 2019).

Na Figura 2 os casos notificados de TA foram classificados de acordo com a evolução dos infectados em cada uma das regiões do país. Os números presentes no gráfico, porém, não representam o total de casos, pois 385 notificações $(12,9 \%)$ estavam com esta informação em branco ou foram ignoradas e em 69 casos (2,3\%) houve óbito por outra causa. 
Figura 2. Evolução dos casos notificados de Tétano acidental no Brasil por região de notificação no período de 2009 a 2019.



Fonte: Dados da pesquisa (2020).

Observou-se que, apesar do alto percentual de cura, a taxa de letalidade do TA no Brasil é expressiva, visto que 38,1\% dos indivíduos contaminados vieram a óbito. Percebe-se, ainda, que um menor número de casos não está associado a uma taxa de letalidade menos acentuada. Exemplo disso é a região Norte, que apesar de apresentar o segundo menor número de contaminados, possui a maior taxa de letalidade $(44,7 \%)$.

A respeito do atendimento destinado aos contaminados, um relato de caso ocorrido em Campo Grande (MS) mostra a imprescindibilidade de um paciente com TA ser acompanhado por uma equipe multidisciplinar, visto que em algumas situações os casos da doença podem vir acompanhados de outras enfermidades (Costa et al., 2019). Ainda quanto à qualidade do atendimento prestado, destaca-se a importância de o profissional de saúde conhecer os aspectos da doença, bem como o método de abordagem frente ao acometido por TA (Ohama et al., 2019; Santos et al., 2020).

Na Figura 3 os casos de tétano foram estratificados de acordo com a faixa etária e o sexo dos infectados. Os intervalos de idade foram mantidos conforme são oferecidos pelo site do DataSUS. 
Figura 3. Casos notificados de Tétano acidental no Brasil por faixa etária e sexo no período de 2009 a 2019.

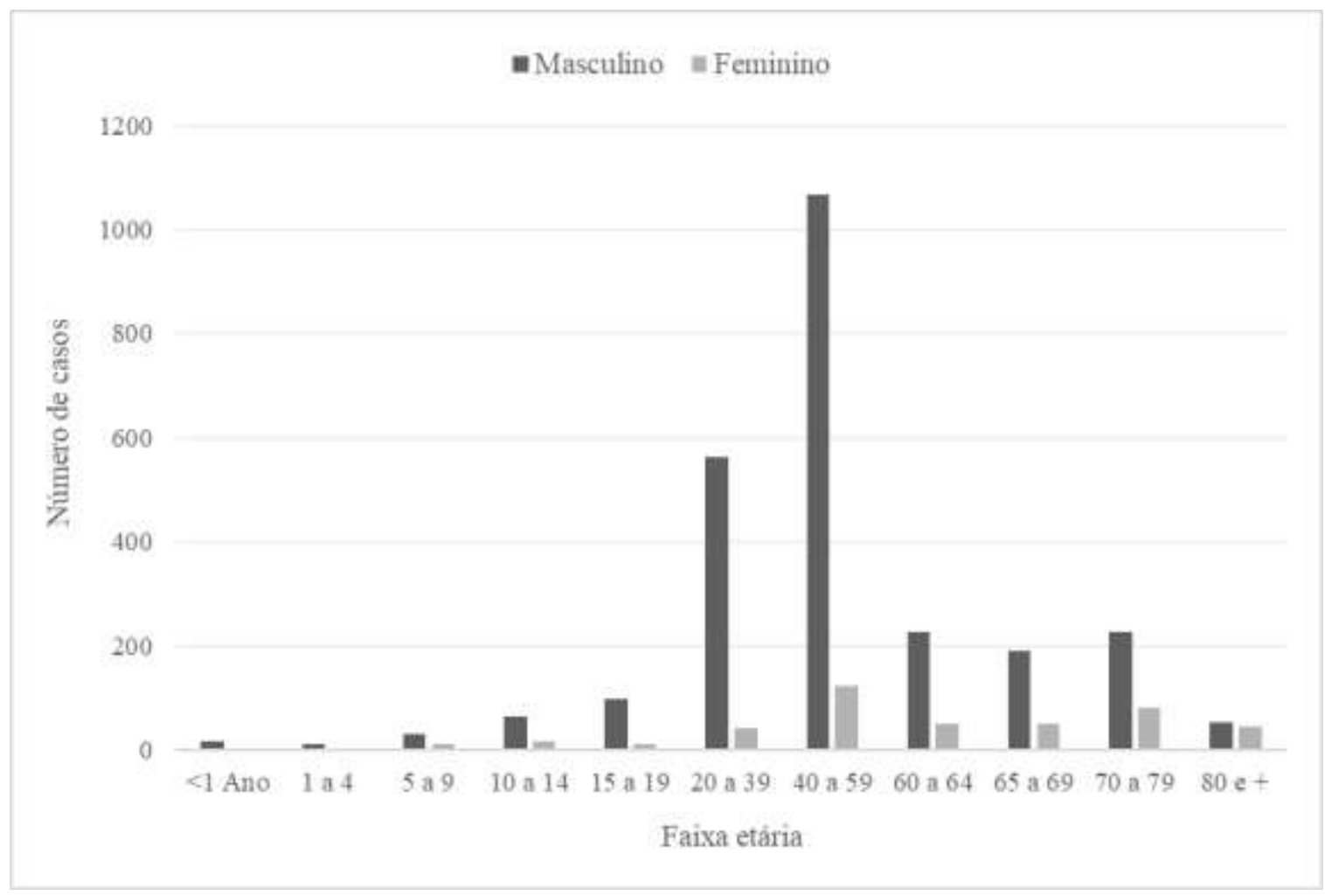

Fonte: Dados da pesquisa (2020).

Observou-se que os contaminados com idade entre 20 e 59 anos são os mais afetados pela doença, representando $60,2 \%$ do total de casos notificados. Estes achados podem estar vinculados à inserção no mercado de trabalho, desconhecimento sobre as formas de prevenção e baixa adesão ou inexistência de esquema vacinal nessa parcela da população.

Em relação à ocupação profissional, o Guia de Imunização da Medicina do Trabalho (2018-2019) reforça a recomendação sobre o recebimento de doses de reforço. Essa recomendação se faz ainda mais importante quando voltada aos indivíduos que realizam funções com alto risco de contaminação, como os trabalhadores rurais (Silva et al., 2020).

Percebe-se, também, que a partir dos 60 anos o número de casos por faixa etária sofre um aumento significativo de $31 \%$. Um dos aspectos que corrobora para esse aumento é o fato de que, com o passar dos anos, os níveis de anticorpos contra o TA decaem e esses indivíduos passam a ter imunidade protetora reduzida (Ministério da Saúde, 2018).

A população de crianças e adolescentes, por outro lado, concentra as menores taxas de casos notificados. Esses resultados podem estar vinculados ao PNI que, segundo dados do DataSUS, apresenta maior cobertura vacinal entre crianças do que entre adultos. Outro fator que pode estar relacionado a esse cenário é a inserção da criança na escola, que tem papel fundamental na disseminação de conhecimento para a prevenção de doenças. A exemplo disso, pode-se citar o Programa Saúde na Escola (PSE), ferramenta muito importante para promoção de saúde entre crianças e jovens da rede pública de ensino (Lopes et al., 2018; Jacob et al., 2019).

Apesar desse contexto positivo em que as crianças se encontram ao serem comparadas com os adultos, nos últimos anos tem-se registrado diminuição na cobertura vacinal infantil. Uma pesquisa revelou aumento significativo no número de óbitos de menores de 1 ano por difteria, tétano e coqueluche, doenças que poderiam ser prevenidas com a vacina pentavalente (Sousa, 2018).

Ao calcular a média de idade de homens e mulheres acometidos por TA, identificou-se, por meio de um Teste T de duas médias independentes, que a média de idade dos homens, 47,7 anos, é significativamente menor que a média de idade das 
mulheres, 55,7 anos (nível de significância de 0,05 e p< 0,0001). Parte das mulheres, quando grávidas e com idade menos avançada, passam pelo acompanhamento pré-natal, em que são vacinadas contra o TA (Santos et al., 2017). Esse pode ser um dos motivos pelos quais elas, se comparadas aos homens, são acometidas mais tardiamente pela doença.

Na Figura 4 os casos de tétano estão estratificados de acordo com a escolaridade dos contaminados. Os números presentes no gráfico, porém, não representam o total de notificações, pois 1.390 (46,6\%) estavam em branco ou foram ignoradas e em 45 casos (1,5\%) a estratificação em questão não era aplicável.

Figura 4. Casos notificados de Tétano acidental no Brasil por escolaridade no período de 2009 a 2019.

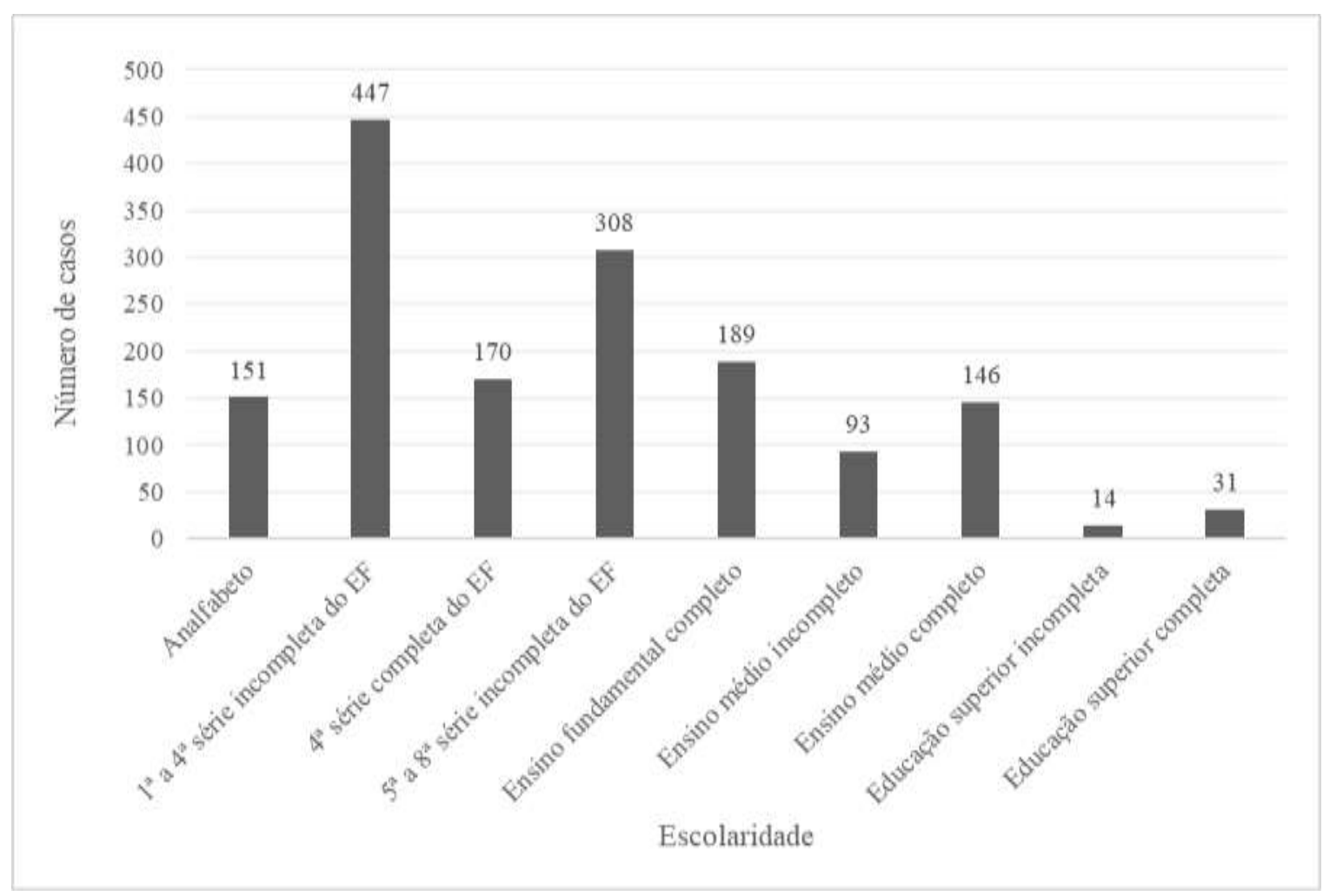

Fonte: Dados da pesquisa (2020).

Observou-se que o maior número de casos $(28,9 \%)$ se concentra entre os infectados que não completaram os estudos da $1^{\mathrm{a}}$ a $4^{\mathrm{a}}$ série do ensino fundamental. Nesse contexto, a Organização Pan-Americana de Saúde (OPAS) (2021) em sua estratégia integral e integradora para o fornecimento de serviços de saúde escolar "Escolas promotoras de saúde" afirma que a escolaridade e a qualidade de vida estão diretamente ligadas à saúde dos indivíduos.

O início do trabalho de conscientização sobre as medidas de profilaxia do tétano deve ocorrer principalmente em idade escolar (Matias et al., 2019). É nítida, portanto, a importância do profissional de enfermagem como propagador da educação em saúde, tendo em vista a carência de conhecimento da população quanto às VCTT’s e demais medidas de prevenção do TA.

Percebe-se, também, que existe uma relação entre o nível de escolaridade dos contaminados e o número de casos, visto que pessoas com ensino médio completo, ensino superior completo ou incompleto representam apenas 12,3\% das 1549 notificações. Um maior nível de escolaridade, permite que as pessoas optem por trabalhar em ambientes menos expostos e mais seguros, onde as chances de contaminação são menores, além de oferecer maior conhecimento sobre a necessidade do recebimento de doses de reforço contra tétano e outras doenças.

Na Figura 5 os casos de TA estão estratificados de acordo com o ano do $1^{\circ}$ sintoma. 
Figura 5. Casos notificados de Tétano acidental no Brasil por ano do primeiro sintoma no período de 2009 a 2019.



Fonte: Dados da pesquisa (2020).

Observou-se, por meio da média anual de 269,9 casos e do desvio padrão de 45,3, que o número de notificações em todos os anos pode ser considerado usual.

Ao comparar o cenário atual com o de décadas passadas, percebe-se que o número de casos da doença tem diminuído significativamente. Na década de 1990 a média foi de 1.000 casos por ano, enquanto na década de 2.000 foi de 460 casos (Ministério da Saúde, 2019).

No período analisado, a partir do ano de 2011 o país registrou queda contínua nas notificações de TA. Realizou-se, então, uma regressão linear simples considerando os casos de TA dos anos de 2011 a 2019 e obteve-se a equação de regressão y=346,81-16,72x, com coeficiente de correlação de -0,9652. Esta regressão resultou na projeção de que, em 2020 e 2021, ocorrerão, respectivamente, 179,6 e 162,9 notificações de casos de tétano acidental no Brasil, com coeficiente de determinação de 93,16\%. Essa queda no número de casos pode estar vinculada à chegada das VCTT's no país na década de 50 (Ministério da Saúde, 2018).

Em comparação com países como os Estados Unidos, onde foram registrados, dentro do período de 2009 a 2017 , apenas 264 casos de TA (Faulkner \& Tiwari, 2020), percebe-se que, mesmo com a diminuição dessas notificações no Brasil, a doença ainda é um problema de saúde pública, uma vez que entre 2009 e 2019 ocorreram um total de 2984 casos.

\section{Considerações Finais}

No Brasil, a região Nordeste apresenta o maior número de casos de TA e a região Norte apresenta o menor índice de cura. Os indivíduos do sexo masculino, com idade entre 20 e 59 anos e baixa escolaridade são os mais afetados pela doença. A partir do ano de 2011 ocorreu uma diminuição no número de notificações.

Apesar da queda no número de casos e da existência de métodos de prevenção capazes de controlar o TA, a quantidade de pessoas acometidas pela doença, no país, ainda é significativa. Acredita-se que isso se deve a uma série de 
fatores, dentre os quais estaria a falta de informação da população e a inacessibilidade dos serviços de saúde. O estudo apresenta como limitações a existência de subnotificações e fichas preenchidas de forma incompleta e/ou inadequada e com pouca exatidão, como nos casos de faixa etária. Recomenda-se a produção de estudos científicos atuais sobre o perfil epidemiológico dos casos de tétano acidental como forma de agregar conhecimento e fortalecer ações de prevenção.

Frente aos achados desta pesquisa é necessário ampliar o conhecimento dos brasileiros acerca das formas de prevenção da doença, por meio da oferta de campanhas de vacinação eficientes e desenvolvimento ampliado do trabalho de enfermagem ligado à educação em saúde, abrangendo todas as idades educacionais e trabalhistas. Ademais, gestores e enfermeiros devem buscar desenvolver estratégias de promoção da saúde e prevenção do TA, oferecendo um acolhimento qualificado para indivíduos que possuem maior risco de contaminação pela doença. Outra prática que demanda atenção é a identificação das ocorrências de TA dentro do SUS, uma vez que um caso notificado com qualidade e detalhamento facilita o conhecimento e as ações de controle da doença.

\section{Referências}

Albuquerque, M. V. D., Viana, A. L. D. Á., Lima, L. D. D., Ferreira, M. P., Fusaro, E. R., \& Iozzi, F. L. (2017). Desigualdades regionais na saúde: mudanças observadas no Brasil de 2000 a 2016. Ciência \& Saúde Coletiva, 22(4), 1055-1064. https://doi.org/10.1590/1413-81232017224.26862016

Costa, S. K. M. D., Pereira, C., Cardozo, C. M. L., Bandeira, L. P., Souza, M. P. D., \& Roriz, N. F. (2019). Atenção multidisciplinar no manejo do tétano acidental em adulto: um relato de experiência. Anais do VII Simpósio Multiprofissional em Saúde: Desafios e Avanços na Assistência à Saúde.

Dantas, M. N. P., Souza, D. L. B. D., Souza, A. M. G. D., Aiquoc, K. M., Souza, T. A. D., \& Barbosa, I. R. (2021). Fatores associados ao acesso precário aos serviços de saúde no Brasil. Revista Brasileira de Epidemiologia, 24, e210004. https://doi.org/10.1590/1980-549720210004

Faulkner, A. E., \& Tiwari, T. S. P. (2020) Manual for the Surveillance of Vaccine-Preventable Diseases. Atlanta: Centers for Disease Control and Prevention, cap.16. https://www.cdc.gov/vaccines/pubs/surv-manual/chpt16-tetanus.html.

Hiester, V. M. (2019). Adesão de trabalhadores à imunização ocupacional: estudo em uma empresa de Santa Cruz do Sul/RS [Trabalho de Conclusão de Curso, Universidade de Santa Cruz do Sul].

Jacob, L. M. D. S., Melo, M. C. D., Sena, R. M. D. C., Silva, I. J. D., Mafetoni, R. R., \& Souza, K. C. S. D. (2019). Ações educativas para promoção da saúde na escola: revisão integrativa. Saúde e Pesquisa, 12(2), 419-426. 10.17765/2176-9206.2019v12n2p419-426

Lopes, I. E., Nogueira, J. A. D., \& Rocha, D. G. (2018). Eixos de ação do Programa Saúde na Escola e Promoção da Saúde: revisão inte grativa. Saúde em Debate, 42(118), 773-789. https://doi.org/10.1590/0103-1104201811819

Matias, F. C., Moura, L. R. D., Albuquerque, M. E. L., \& Santos, M. A. A. D. (2019). Práticas pedagógicas: panfletagem como ferramenta de ensino aprendizado sobre o tétano. Anais do VI Encontro Internacional de Jovens Investigadores.

Ministério da Saúde. (2007). Sistema de Informação de Agravos de Notificação - Sinan: normas e rotinas (2a ed.). Secretaria de Vigilância em Saúde, Departamento de Vigilância Epidemiológica. http://portalsinan.saude.gov.br/images/documentos/Aplicativos/sinan_net/Manual_Normas_e_ Rotinas_2_edicao.pdf

Ministério da Saúde. (2018). Boletim epidemiológico: Situação epidemiológica do tétano acidental no Brasil, 2007-2016 (Vol. 49). Secretaria de Vigilância em Saúde. https://portalarquivos2.saude.gov.br/images/pdf/2018/junho/11/2017-041-Tetano-publicacao.pdf

Ministério da Saúde. (2019). Guia de Vigilância em Saúde: volume único (3a ed.). Secretaria de Vigilância em Saúde, Coordenação-Geral de Desenvolvimento da Epidemiologia em Serviços. https://portalarquivos2.saude.gov.br/images/pdf/2019/junho/25/guia-vigilancia-saude-volume-unico-3ed.pdf

Ministério da Saúde. (2021). Programa Nacional de Imunizações. https://portalarquivos.saude.gov.br/campanhas/pni/

Ohama, V. H., Bezerra, A. M., Castro, E. F. D., \& Sprovieri, S. R. S. (2019). Tétano acidental em adultos: uma proposta de abordagem inicial/Accidental tetanus in adults: an initial approach proposal. Arquivos Médicos dos Hospitais e da Faculdade de Ciências Médicas da Santa Casa de São Paulo, 64(2), 120124. https://doi.org/10.26432/1809-3019.2019.64.2.120

Organização Mundial da Saúde. (2017). Vacinas contra o tétano: documento de posição da OMS, fevereiro de 2017 (n.6). https://www.who.int/wer/2017/wer9206/en/

Organização Pan-Americana da Saúde. (2021). Escolas Promotoras de Saúde. https://www.paho.org/bra/index.php?option=com_content\&view=article\&i $\mathrm{d}=588$ :esc olas-promotoras-de-saude $\&$ Itemid=685.

Santos, C. B., Pereira, D. C., Ferreira, R. B. S., \& Fonseca, E. D. O. S. (2020). Nursing process for patients diagnosed with accidental tetanus. Revista Prevenção de Infecção e Saúde, 6. https://doi.org/10.26694/repis.v6i0.10038

Santos, T. M. M. G. D., Abreu, A. P. D. S. B., \& Campos, T. G. (2017). Avaliação dos registros no cartão de pré-natal da gestante. Rev. enferm. UFPE On Line, 2939-2945. 10.5205/reuol.11007-98133-3-SM.1107sup201715 
Research, Society and Development, v. 10, n. 5, e20010514877, 2021

(CC BY 4.0) | ISSN 2525-3409 | DOI: http://dx.doi.org/10.33448/rsd-v10i5.14877

Silva, C. P. D., Badaró, F. S. D. S., \& Avena, K. D. M. (2020). Tétano Acidental: Perfil clínico-epidemiológico de pacientes internados em hospital de referência de Salvador/Bahia, de 2016-2018. Brazilian Journal of Health Review, 3(4), 7373-7388. https://doi.org/10.34119/bjhrv3n4-012

Sociedade Brasileira de Imunizações \& Associação Nacional da Medicina do Trabalho. (2019). Guia de Imunização SBIM/ANAMT-Medicina do Trabalho. https://sbim.org.br/images/files/guia-sbim-anamt-medicina-trabalho-2018-2019-180730b-web.pdf.

Sousa, B. S. D. Q. (2018). Epidemiologia dos casos de óbitos pelas doenças imunopreviníveis: difteria, tétano e coqueluche em crianças menores de 1 ano no Brasil entre 2001 a 2016. [Trabalho de Conclusão de Curso, Centro Universitário de Brasília].

Tortora, G. J., Case, C. L., \& Funke, B. R. (2017). Microbiologia (12a ed.). Artmed Editora. 